ISBN: 978-93-86878-17-5

12th PATTAYA International Conference on Chemical, Agricultural, Biological and Environmental

Sciences (PCABES-18)

Pattaya (Thailand) May 31-June 2, 2018

\title{
Numerical Study of Exogenous Type Microbial Depolymerization Process
}

\author{
Masaji Watanabe ${ }^{1}$ and Fusako Kawai ${ }^{2}$ \\ ${ }^{1}$ Graduate School of Life and Environmental Science, Okayama University, Japan \\ ${ }^{2}$ Center for Fiber and Textile Science, Kyoto Institute Technology, Japan
}

\begin{abstract}
This study demonstrates the validity of assumption that liberated monomers are converted directly to cells in exogenous type microbial depolymerization processes. Experimental outcomes were incoorporated into inverse analysis of a time factor and a molecular factor of a degradation rate. Once those factors were obtained, microbial depolymerization processes was simulated. The mathematical model is described, and numerical techniques are illustrated with numerical results.
\end{abstract}

Keywords: Biodegradation, Polyethylene glycol, Mathematical model, Numerical simulation, Inverse problem

\section{Introduction}

In an exogenous type microbial depolymerization process, molecules liberate monomer units from their terminals to reduce sizewize. Polyethylene (PE) and polyethylene glycol (PEG) are depolymerizable in exogenous type microbial depolymerization processes. Microbial depolymerization processes of PEG have been well documented. Utilization of PEG of average molecular weight 20000 by Psedonomas aeruginosa was reported [1]. Anaerobic bacteria isolated from sludge of a municipal anaerobic digester degraded PEG 20000 [2]. Efficient biodegradation of PEG by Pseudomonas stutzeri was documented [3]. Microbial depolymerization processes of PE and PEG have been studied numerically. A mathematical model was proposed and numerical techniques were developed in studies of biodegradation of PE [4]. Those numerical techniques were reapplied to microbial depolymerization process of PEG [5].

Unlike exogenous type depolymerization processes, random breakdown of molecules is the essential factor of endogenous type depolymerization processes. Polyvinyl alcohol (PVA) and polylactic acid (PLA) are endogenously depolymerizable polymers. A mathematical model was proposed and numerical techniques were developed in studies of an enzymatic degradation process of PVA [6]. Those numerical techniques were reapplied to an enzymatic hydrolysis of polylactic acid (PLA) [7]. Techniques originally developed for endogenous type processes were replied to exogenous type processes of PE and PEG [8].

This study revisited an exogenous type microbial depolymerization process of PEG. Experimental outcomes were obtained by cultivation of microbial consortium E-1 in culture media, in which PEG was the only carbon source. Those experimental results were incorporated into a computational analysis. Inverse problems for a molecular factor and a time factor of a degradation rate were solved. Once those factors were obtained, entire biodegradation process of PEG was simulated. 


\section{Modeling Of Exogenous Type Microbial Depolymerization Process}

Let $\mathrm{w}(\mathrm{t}, \mathrm{M})[\mathrm{mg}]$ be the weight distribution of a polymer with respect to the molecular weight $\mathrm{M}$ at time $\mathrm{t}$, and let $\mathrm{v}(\mathrm{t})[\mathrm{mg}]$ be the total weight of polymer molecules with molecular weight between $\mathrm{A}$ and $\mathrm{B}$ at time $\mathrm{t}$. The total weight $\mathrm{v}(\mathrm{t})$ over the interval $[\mathrm{A}, \mathrm{B}]$ is expressible in terms of the integral

$$
v(t)=\int_{A}^{B} w(t, M) d M .
$$

The total weight $\mathrm{v}(\mathrm{t})$ of the entire residual polymer at time $\mathrm{t}$ is expressed in terms of the integral

$$
v(t)=\int_{0}^{\infty} w(t, M) d M
$$

Integral (1) serve as an approximation of the integral (2) with appropriate values of A and B. In this study, integral (2) over the infinite interval was replaced with an integral over a finite interval (1) with $A=103.0$ and $\mathrm{B}=104.2$. Similarly, integral with the lower limit 0 was approximated by an integral with the lower limit $\mathrm{A}=103.0$, and an integral with the upper limit $\infty$ was approximated by an integral with upper limit $\mathrm{B}=104.2$.

Suppose that $\sigma(t)$ is the total population of viable cells at time t. System of equations (3), (4) was proposed in previous studies [10 - 15].

$$
\begin{gathered}
\frac{\partial w}{\partial t}=\sigma(t)\left[-\lambda(M) w+c(M) \int_{M}^{\infty} \lambda(K) d(K) w(t, K) d K\right] \\
\frac{d \sigma}{d t}=k\left[-v^{\prime}(t)\right]-h \sigma, \\
c(M)=M e^{\rho M}, d(K)=\frac{\rho e^{-\rho K}}{K\left(1-e^{-\rho K}\right)}, \rho=\frac{\log 2}{L} .
\end{gathered}
$$

Parameter L is the molecular weight of a monomer unit, e,g. PE: L=28 (CH2CH2), PEG: L=44 (CH2CH2O), and $\mathrm{k}$ and $\mathrm{h}$ are positive parameters. Functions $\lambda(M)$ and $\sigma(t)$ are the molecular factor and the time factor of degradation rate, respectively. Equations (2) and (3) lead to the expression

Suppose that $f_{0}(M)$ and $\sigma_{0}^{v^{\prime}(t)=\sigma(t) \int_{0}^{\infty}\left[-\lambda(M) w(t, M)+c(M) \int_{M}^{\infty} \lambda(K) d(K) w(t, K) d K\right] d M}$ are the initial weight distribution and the initial microbial population, respectively. System of equations (3), (4) is accompanied with the initial conditions

$$
\begin{array}{r}
w(0, M)=f_{0}(M), \\
\sigma(0)=\sigma_{0} .
\end{array}
$$

\section{Numerical Techniques for Analysis of Exogenous Type Depolymerization Process}

The initial value problem (3), (4), (6), (7) is solvable provided the molecular factor $\lambda(M)$ and values of parameters $\sigma_{0}, \mathrm{k}$, and $\mathrm{h}$ are prescribed. In order to obtain the function $\lambda(M)$ and values of parameters $\sigma_{0}, \mathrm{k}$, and $h$, consider the change of variables from $t$ to $\tau$

$\tau=\int_{0}^{t} \sigma(s) d s$.
Denote functions corresponding to $\mathrm{w}(\mathrm{t}, \mathrm{M}),{ }^{\sigma(t)}$, and $\mathrm{v}(\mathrm{t})$ by $W(\tau, M), S(\tau)$, and $V(\tau)$, respectively.

Since

$$
\frac{\partial}{\partial \tau}=\frac{\partial}{\partial t} \frac{\partial t}{\partial \tau}=\frac{1}{\sigma(t)} \frac{\partial t}{\partial \tau}
$$


equations (3) and (4) lead to equations

$$
\begin{gathered}
\frac{\partial W}{\partial \tau}=-\lambda(M) W+c(M) \int_{M}^{\infty} \lambda(K) d(K) W(\tau, K) d K, \\
\frac{d S}{d \tau}=k V^{\prime}(\tau)-h
\end{gathered}
$$

respectively. Since

$$
v^{\prime}(t)=\frac{d v}{d t}=\frac{d V}{d \tau} \frac{d \tau}{d t}=V^{\prime}(\tau) \sigma(t)
$$

it follows that

$$
V^{\prime}(\tau)=\frac{\nu^{\prime}(t)}{\sigma(t)}
$$

and that

$$
V^{\prime}(\tau)=\int_{0}^{\infty}\left[-\lambda(M) W(\tau, M)+c(M) \int_{M}^{\infty} \lambda(K) d(K) W(\tau, K) d K\right] d M .
$$

Suppose that $F_{1}(M)$ is the weight distribution for $\tau=T_{1}$, that is,

$$
W\left(T_{1}, M\right)=F_{1}(M),
$$

and that $F_{2}(M)$ is the weight distribution for $\tau=T_{2}$, that is,

$$
W\left(T_{2}, M\right)=F_{2}(M) .
$$

Equation (10), the initial condition (14), and the final condition (15) constitute an inverse problem for $\lambda(M)$, for which the solution of the initial value problem (10), (14) also satisfies the final condition (15).

Numerical techniques were developed for the inverse problem (10), (14), (15) in previous studies. Weight distributions of PEG before after cultivation of microbial consortium E-1 for two days, four days, and seven days, $f 0(M), f 1(M), f 2(M)$, and $\mathrm{f} 3(M)$, were incorporated into analysis. Note that $w(t i, M)=f i(M)(i=0,1,2,3)$, where $\mathrm{t} 0=0, \mathrm{t} 1=2, \mathrm{t} 2=4$, and $\mathrm{t} 3=7$. Weight distributions after cultivation of the microbial consortium $\mathrm{E}-1$ for two days $\mathrm{f1}(\mathrm{M})$ and four days $\mathrm{f} 2(\mathrm{M})$ were assigned to the functions $\mathrm{F} 1(\mathrm{M})$ and $\mathrm{F} 2(\mathrm{M})$, respectively, and the inverse problem (10), (11), (12) was solved numerically for $\mathrm{T} 1=0$ and $\mathrm{T} 2=2$.

Once the function $\lambda(M)$ was obtained, equation (10) was solved for $W(\tau, M)$ with the initial condition

$$
W\left(\tau_{0}, M\right)=f_{0}(M)
$$

where $\tau_{0}=0$, A previous study shows that function $V(\tau)$ is approximated by an exponential function

$$
V(\tau)=v_{0} e^{-\mu \tau} \quad\left(v_{0}=\int_{0}^{\infty} f_{0}(M) d M\right)
$$

Note that $V^{\prime}(\tau)=-\mu v_{0} e^{-\mu \tau}$. In this study, function $V(\tau)$ was approximated by the exponential function (17) with $\mu \approx 0.506$.

Once $V(\tau)$ was obtained, equations $V\left(\tau_{i}\right)=v\left(t_{i}\right) \quad(i=1,2,3)$ were solved numerically, and values $\tau_{1} \approx 0.634, \tau_{2} \approx 2.580$, and $\tau_{3} \approx 6.863$ were obtained.

Suppose that $S\left(\tau, \sigma_{0}, k, h\right)$ is the solution of the equation (11) with initial value ${ }^{\sigma_{0}}$. The change of variables (8) leads to the equation $t=q\left(\tau, \sigma_{0}, k, h\right)$, where 


$$
q\left(\tau, \sigma_{0}, k, h\right)=\int_{0}^{\tau} \frac{d r}{S\left(r, \sigma_{0}, k, h\right)}
$$

Given $m$ pairs of values of $\mathrm{t}$ and $\tau,\left(t_{i}, \tau_{i}\right) \quad(i=1, \ldots, m)$, such that $t_{i}=q\left(\tau_{i}, \sigma_{0}, k, h\right)$, equations for $\sigma_{0}, \mathrm{k}$, and h,

$$
g_{i}\left(\sigma_{0}, k, h\right)=0 \quad(i=1, \ldots, m)
$$

where $g_{i}\left(\sigma_{0}, k, h\right)=q\left(\tau_{i}, \sigma_{0}, k, h\right)-t_{i}$, must hold. In this study, system of equations (18) was solved for $m=3$. The Newton's method was applied to the equation $g_{1}\left(\sigma_{0}, k, h\right)=0$ to obtain the value of $k$ for each fixed pair of values of $\sigma_{0}$ and $h$. That is, the function $\phi\left(\sigma_{0}, h\right)$, such that $g_{1}\left(\sigma_{0}, \phi\left(\sigma_{0}, h\right), h\right)=0$, was obtained. The Newton's method was applied to the equation $g_{2}\left(\sigma_{0}, \phi\left(\sigma_{0}, h\right), h\right)=0$ for evaluation of $h$ for each value of $\sigma_{0}$. That is function $\psi\left(\sigma_{0}\right)$, such that $g_{2}\left(\sigma_{0}, \phi\left(\sigma_{0}, \psi\left(\sigma_{0}\right)\right), \psi\left(\sigma_{0}\right)\right)=0$, was obtained. The iterations were repeated until the residual between two consecutive approximations reduce to a value less than $10^{-8}$. Now the equation $g_{3}\left(\sigma_{0}, \phi\left(\sigma_{0}, \psi\left(\sigma_{0}\right)\right), \psi\left(\sigma_{0}\right)\right)=0$ remains to be solved. Figure 1 shows the curve $\left(\sigma_{0}, g_{3}\left(\sigma_{0}, \phi\left(\sigma_{0}, \psi\left(\sigma_{0}\right)\right), \psi\left(\sigma_{0}\right)\right)\right)$. Figure 1 shows that there is a solution of the equation

$$
g_{3}\left(\sigma_{0}, \phi\left(\sigma_{0}, \psi\left(\sigma_{0}\right)\right), \psi\left(\sigma_{0}\right)\right)=0
$$

in the interval $[0.12,0.13]$. The bisection method was applied to the equation (19), and values of the parameters $\sigma_{0} \approx 0.127, k \approx 0.00380$, and $h \approx 0.0821$ were obtained. Figure 2 and shows the curve $(t, \tau)=\left(q\left(\tau, \sigma_{0}, k, h\right), \tau\right)$ for those values of $\sigma_{0}, k$, and $h$.

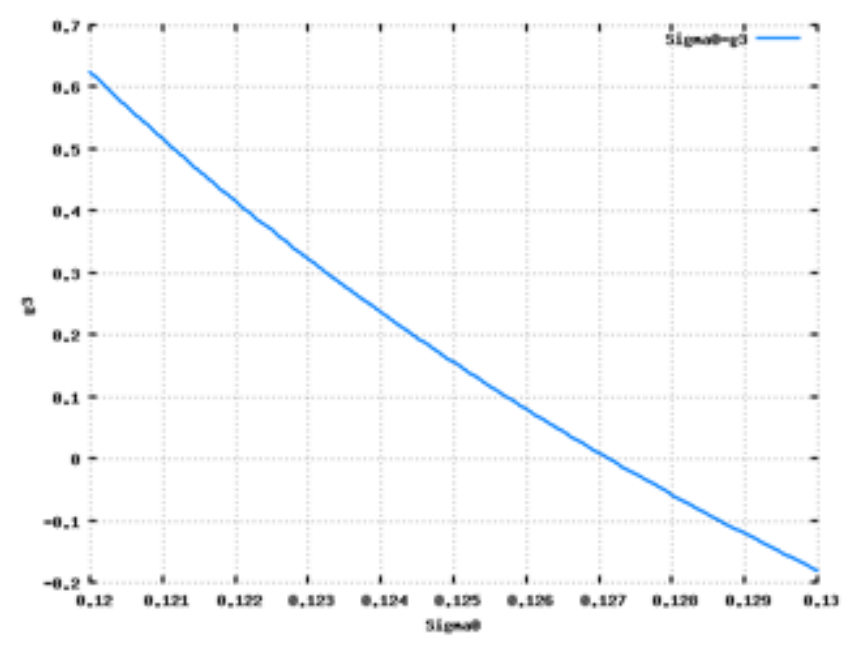

Fig. 1: Curve $\left(\sigma_{0}, g_{3}\left(\sigma_{0}, \phi\left(\sigma_{0}, \psi\left(\sigma_{0}\right)\right), \psi\left(\sigma_{0}\right)\right)\right)$. 


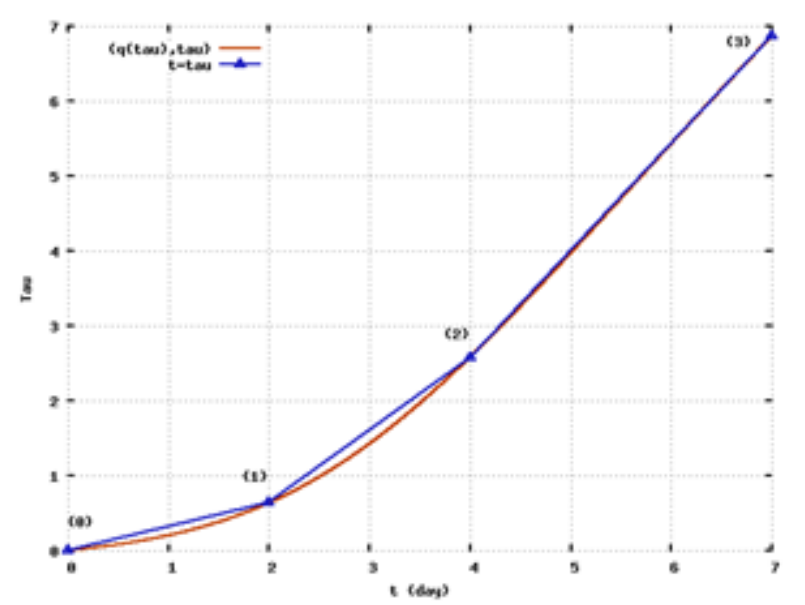

Fig. 2: Curve $(t, \tau)=\left(q\left(\tau, \sigma_{0}, k, h\right), \tau\right)$.

Once the molecular factor $\lambda(M)$ and the time factor $\sigma(t)$ of the degradation rate were obtained, initial value problem (3), (6) was solved numerically. Figure 3 shows numerical results and experimental results for the weight distribution before and after cultivation of the microbial consortium E-1 for two days, four days, and seven days. Figure 4 shows values of optical density OD 630, $O_{0}, O_{1}, O_{2}$, and $O_{3}$ at t0 = 0, t1 = 2, t2 = 4, and $\mathrm{t} 3=7$, and the OD conversion of the microbial population by ratio of areas, that is, $(t, z(t))$ where $z(t)=\sigma(t) / S_{A} * O_{A}[15]$. Here $S_{A}$ and $O_{A}$ are defined by

$$
S_{A}=\int_{t_{0}}^{t_{3}} \sigma(t) d t,
$$

$O_{A}=\frac{O_{0}+O_{1}}{2}\left(t_{1}-t_{0}\right)+\frac{O_{1}+O_{2}}{2}\left(t_{2}-t_{1}\right)+\frac{O_{2}+O_{3}}{2}\left(t_{3}-t_{2}\right)$, are areas of regions in the plane

$$
\left\{(t, x): t_{0} \leq t \leq t_{1}, 0 \leq x \leq \sigma(t)\right\},\left\{(t, x): t_{0} \leq t \leq t_{1}, 0 \leq x \leq O(t)\right\},
$$

respectively, where $O(t)$ is the piecewise linear function

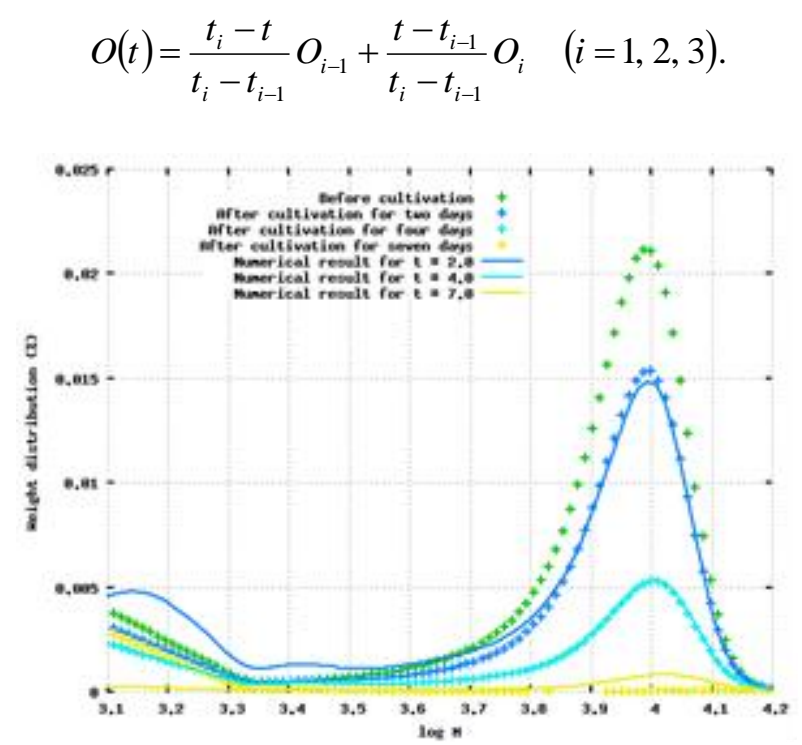

Figure 3: Figure 4: Experimental results and numerical results of weight distribution before and after cultivation of microbial consortium E-1 for two days, four days and seven days. 


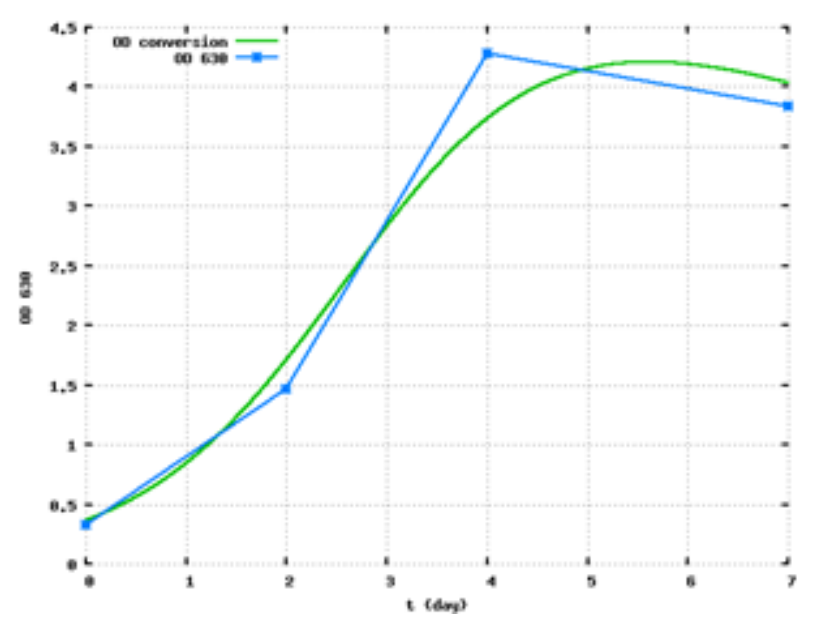

Figure 4: OD 630 and OD conversion of $\sigma(t)$.

\section{Discussion}

Microorganisms utilize polymer molecules as the carbon source in exogenous type microbial depolymerization processes,. The consumption of monomer units by microorganisms is converted to cells, and is the total amount of monomer unit consumed by the microorganisms per unit time. Equation (4) is based on the fact that this amount is converted to the increase of cells.

In a previous study [12], equation (4) was applied to a set of profiles of residual PEG. In recent studies, equation was applied to the set of weight distributions. The Newton's method in conjunction with the bisection method was applied to the system (18). The residual PEG was assumed to be an exponential function , where the value of was obtained from a numerical solution of initial value problem (10), (16) [14]. In this study the Newton's method was applied for numerical solutions of equations, and the bisection method was applied for a numerical solution of the equation. Figure 3 shows numerical results and experimental results concerning the weight distributions after cultivation of the microbial consortium E-1 for two days, four days and the seven days. Acceptable agreements are shown between the numerical results and the experimental results of weight distributions after cultivation for two days and four days, and those numerical results show that our techniques are applicable to inverse analysis with a set of weight distributions.

The optical density accounts for viable cells as well as inviable cells. The resemblance between the OD 630 outcomes and the conversion of microbial population indicates that the majority of cells are viable in the first seven days of coultivation..

\section{Acknowledgment}

The authors thank Ms. Y. Shimizu for her technical support. This work was supported by JSPS KAKENHI Grant Number 16K05276.

\section{References}

[1] J. R. HAINES AND M. ALEXANDER, Microbial Degradation of Polyethylene Glycol, APPLIED MICROBIOLOGY, May 1975, p. 621-625.

[2] DARYL F. DWYER AND JAMES M. TIEDJE, Degradaton of Ethylene Glycol and Polyethylene Glycols by Methanogenic Consortia, APPLIED AND ENVIRONMENTAL MICROBIOLOGY, July 1983. p. 185-190. 
[3] NURIA OBRADORS AND JUAN AGUILAR, Efficient Biodgradation of High-Molecular-Weight Polyethylene Glycols by Pure Cultures of Pseudomonas stutzeri, APPLIED AND ENVIRONMENTAL MICROBIOLOGY, Aug. 1991. p. 2383-2388.

[4] Masaji Watanabe, Fusako Kawai, Masaru Shibata, Shigeo Yokoyama, Yasuhiro Sudate, Shizue Hayashi, Analytical and computational techniques for exogenous depolymerization of xenobiotic polymers, Mathematical Biosciences 192 (2004) 19-37.

https://doi.org/10.1016/j.mbs.2004.06.006

[5] M. Watanabe and F. Kawai, Effects of microbial population in degradation process of xenobiotic polymers, In P. Howlett, M. Nelson, and A. J. Roberts, editors, Proceedings of the 9th Biennial Engineering Mathematics and Applications Conference, EMAC-2009, vol 51 ANZIAM J., pp C682--C96, September 2010 http://journal.austms.org.au/ojs/index.php/ANZIAMJ/article/view/2433

[6] Masaji Watanabe, Fusako Kawai, Mathematical modelling and computational analysis of enzymatic degradation of xenobiotic polymers, Applied Mathematical Modelling 30 (2006) 1497-1514

https://doi.org/10.1016/j.apm.2005.12.011

[7] Masaji Watanabe, Fusako Kawai, Sadao Tsuboi, Shogo Nakatsu, Hitomi Ohara, Study on Enzymatic Hydrolysis of Polylactic Acid by Endogenous Depolymerization Model, Macromolecular Theory and Simulations 16 (2007) 619-626 https://doi.org/10.1002/mats.200700015.

[8] Masaji Watanabe, Fusako Kawai, Simulation for microbial depolymerization processes of polyethylene glycol, Proceedings of the 2013 International Conference on Advances in Intelligent Systems in Bioinformatics, Editors: Dr. Ford Lumban Gaol, Dr. Benfino Soewito, Prof. Dr. Mohamed Bououdina, Prof. Dr. Mu-Song Chen, intel-13, Advances in Intelligent Systems Research, Atlantis Press, February 2014, 71 - 76. ISBN: 978-94-6252-000-4, ISSN: 1951-6851 http://www.atlantis-press.com/php/pub.php?publication=intel-13

[9] Masaji Watanabe, Fusako Kawai, Numerical Techniques for Inverse Problems from Modeling of Microbial Depolymerization Processes, International Journal of Applied Engineering Research ISSN 0973-4562 Volume 11, Number 8 (2016) pp 5461-5468. http://www.ripublication.com/ijaer16/ijaerv11n8_18.pdf

[10] Masaji WATANABE and Fusako KAWAI, Simulation of Microbial Depolymerization Process with Exponential Consumption of Carbon Source, 2016 International Conference on Computer, Mechatronics and Electronic Engineering (CMEE 2016), DEStech Publications, Inc., Lancaster, Pennsylvania, U.S.A., 2016, 426-431, ISBN: 978$1-60595-406-6$

[11] Masaji Watanabe, Fusako Kawai, Numerical Techniques for Simulation of Microbial Depolymerization Process with Time Factor of Degradation Rate, Submitted.

[12] M Watanabe and F Kawai, Computational analysis for biodegradation of exogenously depolymerizable polymer, 2017 the 5th International Conference on Mechanical Engineering, Material Science and Civil Engineering, December, 2017, Kuala Lumpur, Malaysia, 012015, IOP Conference Series: Materials Science and Engineering, Published online: 6 April 2018. IOP Publishing http://iopscience.iop.org/issue/1757-899X/324/1

[13] FUSAKO KAWAI AND SHOGO ENOKIBARA, Symbiotic Degradation of Polyethylene Glycol (PEG) 20,000Phthalate Polyester by Phthalate Ester Ester- and PEG 20,000-Utilizing Bacteria, JOURNAL OF FERMENTATION AND BIOENGINEERING, Vol. 82, No.6, 575-579, 1996.

https://doi.org/10.1016/S0922-338X(97)81255-X

[14] MASAJI WATANABE, FUSAKO KAWAI, APPLICATION OF THE NEWTON'S METHOD AND BISECTION METHOD TO THREE PARAMETER PROBLEM FOR MICROBIAL DEPOLYMERIZATION PROCESS, Proceedings, ISER INTERNATIONAL CONFERENCE, 1st - 2nd April, 2018| Venue: Kuala Lumpur, Malaysia

[15] M Watanabe and F Kawai, Computational study of bacterial depolymerization process of xenobiotic polymer, Submitted to International Conference on Engineering Physics and Optoelectronic Engineering (ICEPOE 2018). 